\title{
Trauma de vena cava en pacientes politraumatizados: Experiencia en el Hospital Dr. Sótero del Río
}

\author{
Juan Pablo Ramos P. ${ }^{1}$, Analía Zinco A. ${ }^{1}$, Pablo Ottolino L. ${ }^{1}$, \\ Carolina Muñoz A. ${ }^{1}$ y Alfonso Díaz F. ${ }^{1}$
}

\section{Vena cava injury in politraumatized patients}

Introduction: For its retroperitoneal location, vena cava injuries are infrequent, however, occurring to a patient with multiple intra-abdominal injuries and in critical condition. Aim: To describe the experience of polytraumatized patients with VCI who underwent surgical resolution in the Dr. Sotero del Rio hospital. Materials and Method: Retrospective analysis of 36 polytraumatized patients that showed VCI during the surgical intervention within 2010 and 2017. Results: The series was composed of 36 VCI patients. $88.8 \%$ are men, in $97.2 \%$ the mechanism of trauma is penetrating. Within the vascular anatomical levels, the most frequently injured are the infra-renal cava $(41.6 \%)$ suprarenal $(25 \%)$, yuxta-renal $(22.2 \%)$ retro-hepatic and intrathoracic $(5.5 \%$ each). The most frequent associated injured structures were the small intestine (38.8\%) other vessels of large caliber (36.1\%) and kidney (30.5\%). In relation to the type of surgical resolution, $15.6 \%$ had a vena cava ligature and $83.3 \%$ simple raffia. In $33.3 \%$, a thoracotomy was required for vascular control, two of them were resuscitative procedures. The average of hospitalization was 19.8 days. The mortality was $33.3 \%$. Conclusions: According to what presented, the high mortality of VCI was found in the group of patients with hemodynamic instability and the number of associated injuries; specially if other large vessels are involved. To optimize the survival, the efforts should be focused on a fast prehospital transfer and the training for surgeons who face this type of injury.

Key words: vena cava injury; large vessels; treatment.

\section{Resumen}

Introducción: Por su ubicación retroperitoneal, las lesiones de vena cava (LVC) son infrecuentes, presentándose en el contexto de un paciente con múltiples lesiones intraabdominales y en estado crítico. Objetivo: Describir la experiencia de pacientes politraumatizados con LVC sometidos a resolución quirúrgica en Hospital Dr. Sotero del Río. Materiales y Método: Análisis retrospectivo de 36 pacientes politraumatizados que dentro de la intervención quirúrgica se evidenció LVC en un período comprendido entre el 2010 y 2017. Resultados: La serie estuvo compuesta por 36 pacientes, con LVC. El 88,8\% son hombres, en el $97,2 \%$ el mecanismo de trauma es penetrante. Dentro de los niveles anatómicos vasculares más frecuentemente lesionados está la cava infrarrenal $(41,6 \%)$, suprarrenal $(25 \%)$, yuxtarrenal $(22,2 \%)$ y la retrohepática e intratorácica $(5,5 \%$ cada una). Las estructuras lesionadas asociadas más frecuentes fueron intestino delgado $(38,8 \%)$, otros vasos de gran calibre $(36,1 \%)$ y riñón $(30,5 \%)$. Dentro del tipo de resolución quirúrgica, al $15,6 \%$ se realizó ligadura de vena cava y al $83,3 \%$ rafia simple. En $33,3 \%$ fue necesaria una toracotomía para el control vascular, siendo 2 de estas toracotomías resucitadoras. La media de hospitalización fue de 19,8 días. La mortalidad fue de 33,3\%. Conclusiones: De acuerdo a lo presentado, la alta mortalidad de LVC se encontró en el grupo de pacientes con inestabilidad hemodinámica y número de lesiones asociadas, en especial si involucran otros grandes vasos. Los esfuerzos para optimizar la supervivencia se deberían dirigir al traslado rápido prehospitalario y al entrenamiento de los cirujanos que enfrentan este tipo de lesiones.

Palabras clave: trauma vena cava; grandes vasos; manejo. 


\section{Introducción}

El trauma vascular abdominal continúa siendo una entidad clínica letal, a pesar de los avances en los cuidados prehospitalarios y de reanimación del paciente politraumatizado. A lo largo de los años se mantienen cifras estables de mortalidad que van desde el 20 al $50 \%{ }^{1}$.

Las lesiones de vena cava (LVC) se presentan hasta en un $40 \%$ de las lesiones vasculares abdominales ${ }^{2}$ y por su ubicación retroperitoneal, se presentan en el contexto de un paciente con múltiples lesiones asociadas e inestable hemodinámicamente, lo que determina su alta tasa de mortalidad. El principal mecanismo de trauma es el penetrante y menos del $10 \%$ son por causa de trauma contuso. La vena cava inferior se lesiona en promedio 1 de cada 300 heridas por arma blanca y 1 de cada 50 por arma de fuego $0^{3,4}$. De los pacientes con LVC, un tercio no llegará vivo al centro hospitalario y del resto, un alto porcentaje fallecerá dentro de las primeras $24 \mathrm{~h}$ desde el ingreso, principalmente a causa de complicaciones derivadas del shock hipovolémico y las múltiples lesiones asociadas ${ }^{2}$. La vena cava inferior (VCI) intraabdominal se divide topográficamente en 5 secciones: Infrarrenal, pararrenal (segmento que se extiende $2,5 \mathrm{~cm}$ proximal y distal a las venas renales), suprarrenal, retrohepática y suprahepática, cada una de estas difiere en su acceso, manejo y pronóstico ${ }^{3}$. Por todo lo anteriormente explicado, la LVC representa un desafío permanente para el cirujano en el servicio de urgencias.

El objetivo del presente estudio es exponer la experiencia de cirujanos generales en el manejo de pacientes politraumatizados con lesión de vena cava que ingresan al Hospital Dr. Sótero del Río en el período comprendido entre el año 2010 y 2017.

\section{Materiales y Método}

Estudio descriptivo retrospectivo de una serie de 36 casos de pacientes politraumatizados, que dentro de la laparotomía exploradora se evidenció LVC, en el período comprendido entre los años 2010 y 2017 atendidos en el Hospital Dr. Sótero del Río. Se revisaron los registros de pabellón, seleccionando los pacientes mayores de 15 años sometidos a laparotomía exploratoria por trauma abdominal contuso o penetrante. Se incluyeron variables como signos vitales al ingreso, tiempo preoperatorio, realización de toracotomía, topografía del trauma de vena cava, lesiones asociadas, requerimientos transfusionales, tipo de reparación, tiempo quirúrgico, días de hos- pitalización, reintervenciones y mortalidad. Se presentan los resultados, expresados en distribuciones de frecuencia y porcentaje.

\section{Resultados}

La serie estuvo compuesta por 36 pacientes con una mediana de 28 años (rango 15 - 52 años). De estos el $88,8 \%$ son hombres, en el $52,7 \%$ el mecanismo de trauma es por arma de fuego y se evidenció solo 1 paciente con trauma contuso (Tabla 1).

Solo dos pacientes $(5,5 \%)$ en la serie ingresan en paro cardiorrespiratorio (PCR) y la gran mayoría, el 58,33\% ingresan hemodinámicamente estables como se muestra en la Tabla 2. En 12 pacientes $(33,3 \%)$ fue necesaria una toracotomía para el control vascular, siendo 2 de estas $(5,5 \%)$ de resucitación en la sala de emergencia.

Dentro de los niveles anatómicos vasculares más frecuentemente lesionados se encuentra, en primer lugar, la cava infrarrenal con 15 pacientes $(41,6 \%)$, luego la cava suprarrenal con 9 pacientes $(25 \%)$, seguido de la cava para o yuxtarrenal con 8 pacientes $(22,2 \%)$, y por último la cava retrohepática y la intratorácica con 2 pacientes cada una $(5,5 \%$ respectivamente) (Figura 1).

Al evaluar la relación del segmento anatómico lesionado con respecto a la mortalidad, encontramos que los pacientes con LVC retrohepática el $100 \%$ falleció a diferencia de la menor mortalidad encontrada a nivel yuxtarrenal (25\%) e intratorácico donde no ocurrió mortalidad en este último grupo de pacientes como lo muestra la Tabla 3 y Figura 1.

Tabla 1. Distribución del mecanismo de lesión

\begin{tabular}{|lcc|}
\hline Mecanismo del trauma & n & \% \\
\hline Arma blanca & 16 & 44,4 \\
\hline Arma de fuego & 19 & 52,7 \\
\hline Trauma contuso & 1 & 2,7 \\
\hline
\end{tabular}

Tabla 2. Distribución según signos vitales al ingreso

\begin{tabular}{|lrc|}
\hline Signos vitales al ingreso & n & \% \\
\hline Paro Cardiorrespiratorio & 2 & 5,5 \\
Hipotensión & 13 & 36,1 \\
Normotensión & 21 & 58,33 \\
Taquicardia & 23 & 63,8 \\
\hline
\end{tabular}


ARTíCULO ORIGINAL

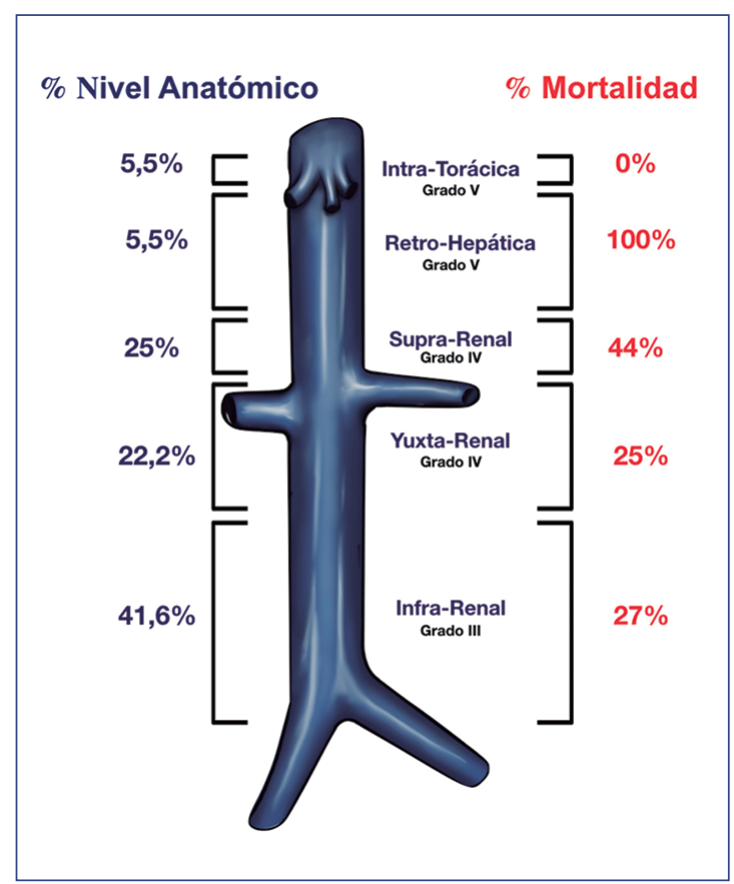

Figura 1. Distribución según nivel anatómico.

El $100 \%$ de los pacientes presentó lesiones asociadas, de las cuales, entre las más frecuentes se encuentran: Intestino delgado en 14 pacientes $(38,8 \%)$, otros vasos de gran calibre como aorta, vasos renales, esplénicos, arteria mesentérica superior e inferior y vena porta, en 13 pacientes $(36,1 \%)$, además de localización torácica como muestra la Tabla 4. El 100\% de los pacientes que presentaron lesión asociada de aorta (6 pacientes) fallecieron, así como también los que tuvieron lesión asociada de venas renales, arteria mesentérica superior y vena esplénica.

Dentro del tipo de tratamiento quirúrgico a 6 pacientes $(16,6 \%)$ se realizó ligadura de vena cava y a 30 pacientes $(83,3 \%)$ se realizó reparación con sutura simple, no se reportaron casos de anastomosis, packing, uso de injertos o shunt (Tabla 5). En 7 pacientes $(19,4 \%)$ el tiempo quirúrgico fue menor a una hora y en 29 pacientes $(80,5 \%)$ fue mayor a una hora. Los días de hospitalización tuvieron un rango entre 4 y 49 días, con una media de 19,8 días. La mortalidad fue de $33,3 \%$ (12 pacientes), de los cuales 6 pacientes fallecieron en el intraoperatorio. Los 30 pacientes restantes de la serie tienen un alto porcentaje de morbilidad, esto en el contexto de presentar un grupo de pacientes grave que requirió manejo intensivo. Estos resultados se muestran en la Tabla 6.
Tabla 3. Distribución según segmento anatómico lesionado y mortalidad

\begin{tabular}{|lccc|}
\hline Segmento anatómico & $\begin{array}{c}\text { N: Pacientes con } \\
\text { lesión }\end{array}$ & $\begin{array}{c}\text { N: Pacientes } \\
\text { fallecidos }\end{array}$ & $\begin{array}{c}\text { (\%) Pacientes } \\
\text { fallecidos }\end{array}$ \\
\hline Intratorácica & 2 & 0 & 0 \\
\hline Retrohepática & 2 & 2 & 100 \\
Suprarrenal & 9 & 4 & 44,4 \\
Yuxtarrenal & 8 & 2 & 25 \\
Infrarrenal & 15 & 4 & 26,6 \\
\hline
\end{tabular}

Tabla 4. Distribución de estructuras lesionadas asociadas a VC

\begin{tabular}{|lcc|}
\hline Estructura lesionada & $\mathbf{n}$ & $\mathbf{\%}$ \\
\hline Intestino delgado & 14 & 38,8 \\
\hline Grandes vasos & 13 & 36,1 \\
\hline Riñón & 11 & 30,5 \\
\hline Hígado & 6 & 16,6 \\
\hline Duodeno & 5 & 13,8 \\
\hline Colon & 4 & 11,1 \\
\hline Bazo & 3 & 8,3 \\
\hline Páncreas & 3 & 8,3 \\
\hline Estómago & 3 & 8,3 \\
\hline Pulmón & 2 & 5,5 \\
\hline Diafragma & 2 & 5,5 \\
\hline Corazón & 2 & 5,5 \\
\hline
\end{tabular}

Tabla 5. Distribución según tipo de reparación quirúrgica

\begin{tabular}{|lcc|}
\hline Tipo de reparación & n & \% \\
\hline Ligadura & 6 & 16,6 \\
Rafia & 30 & 83,3 \\
\hline
\end{tabular}

Tabla 6. Morbilidad de la serie según Clavien-Dindo

\begin{tabular}{|ccc|}
\hline Grado & n & \% \\
\hline I & 5 & 16,6 \\
\hline II & 10 & 33,3 \\
\hline IIIb & 2 & 6,6 \\
\hline IVa & 5 & 16,6 \\
\hline IVb & 2 & 6,6 \\
\hline V & 6 & 20,0 \\
\hline
\end{tabular}




\section{Discusión}

El trauma de forma general afecta en su gran mayoría a hombres jóvenes y particularmente, la LVC no escapa a esta proporción, lo cual fue evidenciado en este estudio y coincide con todos los reportes publicados hasta ahora ${ }^{1-3,5}$. El mecanismo de trauma más frecuente fue por arma de fuego, el cual siempre ha predominado en los reportes de LVC independientemente de la época o ubicación geográfica ${ }^{3}$.

En nuestra serie, $36,1 \%$ de los pacientes llegan a la emergencia inestables hemodinámicamente y $5,5 \%$ llegan en paro cardiorrespiratorio, ameritando en el 33,3\% de los casos toracotomía amplia para control vascular y masaje cardíaco directo, lo cual está asociado con un incremento en la mortalidad descrito en la literatura que señala que $57 \%$ de los pacientes que ameritan toracotomía en la sala de urgencias fallecen, así como también el $25 \%$ de los pacientes con toracotomía realizadas en el intraoperatorio ${ }^{3}$. Lo contrario ocurre con los pacientes que sobreviven a una LVC: ninguno tiene toracotomía en la sala de urgencias y solo un $10 \%$ tiene toracotomía intraoperatoria ${ }^{3,4}$.

La mortalidad global fue de $33,3 \%$, menor que la reportada en otras series que varía del 40 al $88 \%$. Sin embargo, esta cifra no puede ser comparada entre los diferentes centros, al ser una variable multifactorial, en especial al depender de una óptima atención prehospitalaria, la proporción de pacientes que llegan inestables o in extremis y la capacidad o recursos disponibles en cada hospital. Cabe destacar que en esta serie el $100 \%$ de los pacientes fueron intervenidos por cirujanos generales, lo que lleva a pensar en la necesidad de cirujanos especialistas en este tipo de pacientes y aumentar la supervivencia y mejorar morbilidad.

Específicamente en LVC la supervivencia depende del segmento anatómico comprometido, ya que algunos de estos son de muy difícil acceso, lo cual impide un rápido y adecuado control vascular, terminando en la rápida exanguinación del paciente. En nuestra serie el $100 \%$ de los pacientes con lesión de cava retrohepática fallecieron, similar a lo descrito por otros autores ${ }^{3,6,7}$. Seguida de ésta, encontramos $44,4 \%$ de mortalidad en lesiones de vena cava suprarrenal, lo cual también se relaciona a su difícil acceso y campo quirúrgico limitado a la hora de la reparación, sin embargo, la mortalidad en este segmento anatómico fue menor al $78 \%$ reportado en otras series ${ }^{7}$. En la lesión a nivel infrarrenal encontramos una mortalidad de $26,6 \%$, el cual es inferior al $33 \%$ descrito en otras literaturas ${ }^{5}$, proba- blemente a su fácil acceso y rápido control vascular. Por otro lado, el $100 \%$ de los pacientes con LVC intratorácica (segmento intrapericárdico) sobrevivió, lo cual difiere a lo reportado en la literatura la cual describe mayor mortalidad, mientras más proximal es la lesión. Distintas series reportan que solo 1 de 6 pacientes sobreviven a una lesión de vena cava intrapericárdica ${ }^{3}$. Probablemente la alta tasa de supervivencia de este tipo de lesiones en nuestra serie se deba a que estas fueron tratadas desde un principio como heridas penetrantes cardíacas y fueron llevados sin demora a quirófano para toracotomía, control vascular y reparación inmediata; además que este tipo de pacientes si logran sobrevivir hasta llegar al centro de salud, probablemente es porque tienen lesiones pequeñas, fáciles de reparar o se presentan con taponamiento cardíaco que contiene la hemorragia? ${ }^{7}$.

El método de reparación más usado fue la rafia simple, en $83,3 \%$ de los casos. Aunque en esta serie se llevó a cabo la ligadura de vena cava infrarenal, se prefiere este procedimiento para pacientes con shock profundo e inestables hemodinámicamente en los que no es posible realizar una reparación primaria. La ligadura de la cava en cualquier nivel superior al yuxtarrenal está contraindicado, ya que bloquea el retorno venoso renal, lo cual es incompatible con la vida, en estos casos se debe hacer reparación y si el paciente está inestable una alternativa adecuada es el uso de shunt intravascular ${ }^{3,4}$. Todos los pacientes a los cuales se les ligó la vena cava, fue a nivel infrarrenal.

Todos los casos presentaron lesiones asociadas, las cuales agravan el pronóstico, en especial si se encuentra comprometida otra estructura vascular, teniendo una mortalidad del $100 \%$ en nuestra serie cuando se asoció a lesión de aorta, arteria mesentérica superior o venas renales. Esto coincide con todas las series revisadas, las cuales señalan que la asociación de LVC con trauma de aorta y vena porta es universalmente fatal ${ }^{8-10}$. En nuestra serie, el 36,1\% de los pacientes presentó lesión asociada de grandes vasos, mayor a lo encontrado en otras series, que reportan un $10 \%$ de incidencia. La mayor tasa de mortalidad de la serie se encontró en los grupos que llegaron hemodinámicamente inestables, en los que se presentó lesión en la zona retrohepática y los que presentaron lesiones vasculares mayores asociadas.

\section{Conclusiones}

La LVC tiene alta tasa de mortalidad, en especial si se encuentra involucrada la cava retrohepática o suprahepática. Las lesiones de la cava infrarrenal 
son las más comunes, siendo el segmento más fácil de exponer para su reparación. La mayor mortalidad de la serie se encontró en los pacientes inestables al ingreso y con localización retrohepática.

Aunque en el transcurso del tiempo han habido mejoras en cuanto a tecnología y protocolos de reanimación, la mortalidad debida a LVC se ha mantenido estable, por lo que aún se debe enfocar la atención en optimizar los sistemas de atención prehospitalaria, así como también en el entrenamiento de los cirujanos que enfrentan estas lesiones tan devastadoras.

\section{Responsabilidades éticas}

Protección de personas y animales. Los autores declaran que para esta investigación no se han realizado experimentos en seres humanos ni en animales.

Confidencialidad de los datos. Los autores declaran que en este artículo no aparecen datos de pacientes.

Conflicto de Intereses: Ninguno.

\section{Referencias}

1. Rosengart MR, Smith DR, Melton SM, May AK. Prognostic factors in patients with inferior vena cava injuries. Am Surg. 1999;65:849-56.

2. Kuehne J, Frankhouse J, Modrall G, Golshani S, Aziz I, Demetriades D. Determinants of survival after inferior vena cava trauma. Am Surg. 1999; 65:976-81

3. Huerta S, Bui T, Nguyen T, Banimahd F, Porral D. Predictors of mortality and management of patients with traumatic inferior vena cava injuries. The American Surgeon 2006;72;290-6.

4. Buckman RF, Pathak AS, Badellino MM, Bradley KM. Injuries of the inferior vena cava. Surg Clin North Am. 2001;81:143147.

5. Starzl TE, Kaupp HA Jr, Beheler EM, Freeark RJ. Penetrating injuries of the vena cava. Surg Clin N Amer.1963;43:387-400.

6. Van Rooyen PL, Karusseit VO, Mokoena T. Inferior vena cava injuries: A case series and review of the South African experience. Injury 2005;46:71-5.

7. Hansen CJ, Bermadas C, West MA,
Ney AL, Muehlstedt S, Cohen M, et al. Abdominal vena caval injuries: outcomes remain dismal. Surgery 2000;128:572-8.

8. Robert EA, William BF. Injuries to the Inferior Vena Cava. Surg Clin N Amer. 1972;52: 699-710.

9. Paul JS, Webb TP, Aprahamian C, Weiglet JA. Intraabdominal vascular injury: are we getting any better? J Trauma Injury Infect Crit Care 2010;69:1393-7.

10. Asensio JA, Chahwan S, Hanpeter D, Demetriades D, Forno W, Gambaro E, et al. Operative management and outcome of 302 abdominal vascular injuries. Am J Surg. 2000;180:528-34. 